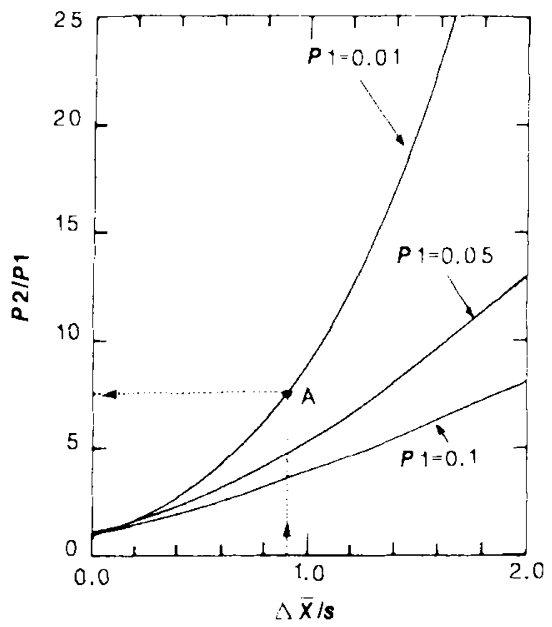

Sensitivity of the frequency of extreme events to changes in the mean (based on an assumed normal distribution and constant standard deviation). Abscissa shows the change in mean $(\triangle X)$ as a multiple of standard deviation (s); ordinate shows the resulting change in the probability of extreme events with initial probabilities $(P 1)$ of $0.1,0.05$ and 0.01 .

inter-annual variability and in year-toyear persistence of climate may either amplify or dampen these influences of a changing mean. These ideas have been developed in more detail by Parry and Carter ${ }^{2}$

This focus on variability and extremes has been examined for shorter timescales by L. O. Mearns, R. W. Katz and S. H. Schneider ${ }^{3}$. Noting that some crops are sensitive to temperature extremes (for example, a few consecutive days over $95^{\circ} \mathrm{F}$ can significantly reduce corn yields in the United States they have used an autoregressive model to calculate the effects of a changed mean on the frequency of daily extremes, specifically daily maxima over $95^{\circ} \mathrm{F}$. The stochastic simulation approach they use is essentially that used in stochastic hydrology, where the technique has been developed to a high degree over the past 20 years. (At least one study ${ }^{4}$ has considered the impact of climatic change on water resources in this way, although the results were rather inconclusive.) Echoing Parry's conclusion, Mearns et al. find that the relationship between changes in mean temperature and changes in the probability of daily extremes is highly non-linear, and that small changes in the mean can sometimes produce relatively large changes in the probabilities of certain events. Their results are sensitive to assumptions regarding changes in variability and changes in day-to-day autocorrelation, but the qualitative conclusions hold even if these parameters are varied.

Changes in the frequency of extreme high (or low) temperatures are of importance not only to agriculture but also, for example, to energy demand and human mortality, so this type of analysis can provide broad insights into the way that climatic change may affect society. It also opens up new avenues for multidisciplinary research into climate impact assessment.

1. Parry, M. L. Climatic Change, Agriculture and Settlement (Dawson, Folkstone, 1978).

2. Parry, M. L. \&arter, T. R. Climatic Change 7, 95 (1985). Mearns, L. O., Katz, R. W. \& Schneider, S. H. J clim. appl. Meteorol. 23, 1601 (1984).

4. Schwarz, H. E in Climate, Climatic Change, and Water Supply 111 (Nat. Acad. Sci., Washington, DC: 1977)

T.M:L. Wigley is in the Climatic Research Unit School of Environmental Sciences, University of East Anglia, Norwich NR4 7TJ, UK.

\title{
Neuropharmacology
}

\section{Super-potent serotonin blockers}

\author{
from Leslie L. Iversen
}

STarting on page 126 of this issue, B. P. Richardson and colleagues from Sandoz describe the discovery of a novel class of serotonin antagonists which offer new insights into the pharmacology of this biologically active indolamine ${ }^{1}$. The new drugs are among the most potent of any type ever described: some act at concentrations as low as $10^{-14} \mathrm{M}$. The compounds, which bear a structural resemblance to both serotonin and cocaine are indoletropanyl or homotropanyl esters. They specifically block the ability of serotonin to excite various peripheral nerves in the autonomic and sensory nervous systems. Furthermore, different compounds in the new series show wide variations in their potency for antagonizing the effects of serotonin on different types of peripheral nerve, suggesting that neural receptors for serotonin (termed $\mathrm{M}$ receptors) comprise a variety of distinct pharmacological sub-classes.

One important role of the M-receptors may be to mediate the painful effects of serotonin released during injury or vascular spasm, by triggering activity in the endings of pain fibres. One of the new antagonists, ICS 205-930, can block the painful effects of serotonin administered locally to human skin and may prove useful in controlling pain in migraine sufferers.

Serotonin (5-hydroxytryptamine, 5-HT) has been known for some forty years, and its pharmacological actions on various tissues have been studied intensively. Gaddum was first to show that the actions serotonin on some smooth muscles can be blocked by the powerful hallucinogen D-lysergic acid diethylamide (DLSD). A number of different receptor classes for serotonin has been suggested in peripheral tissues and in brain. Gaddum and Picarelli ${ }^{2}$ showed that serotonin con-

\section{0 years ago}

THE COMET OF 1472. - M. Celoria of Milan has discussed the elements of the last comet observed by Toscanelli, which is the celebrated one of 1472 , also observed by Regiomontanus, whose description of its path in the heavens enabled Halley to make a rough approximation to its orbit, as he states in his "Synopsis of Cometary Astronomy." The Chinese account of the comet's track contained in the supplement to the great collection of Ma Twan Lin, of which Edouard Biot published a translation in the appendix to the "Connaissance de Temps" for 1846 , enabled Langier to make a further calculation of the orbit, though the somewhat full description of the comet's course amongst the stars is unfortunately very deficient in dates.

From Nature 32 231, 9 July 1885

tracts the intestine by two different mechanisms: a direct action on smooth muscle, mediated by D-receptors, and an indirect action on autonomic nerves to stimulate acetylcholine release, mediated by $\mathrm{M}$-receptors on neurones. More recently, radioligand binding studies have also revealed two classes of binding sites, termed 5-HT, and 5-HT, The 5-HT, sites are identified by high affinity binding of serotonin in brain and may mediate contractile responses in some peripheral tissues, particularly stomach. The 5- $\mathrm{HT}_{2}$ sites, which may correspond to the Dreceptors, are found in both brain and periphery; they mediate contractile responses in peripheral blood vessels and aggregation responses of blood platelets to serotonin ${ }^{3}$. These sites are potentially blocked by classical serotonin antagonists: cyproheptadine, cinanserin, methergoline, methysergide and ketanserin; in brain they are identified by using radiolabelled ketanserin as a ligand ${ }^{4}$. Both classes are potently blocked by D-LSD, although it is not known whether this explains LSD's unique psychopharmacology. Neither class is sensitive to the new Sandoz analogues, which appear to be specific for the neural M-receptors.

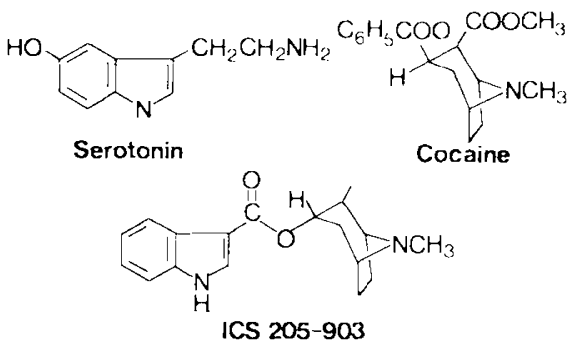

The development of the new compounds stems in part from the discovery by J. R. Fozard ${ }^{5}$, that cocaine is a weak but selective antagonist of neural responses to serotonin. Fozard and his colleagues at the Merrell Dow laboratories in Strasbourg used this discovery to develop a series of selective serotonin antagonists modelled on the cocaine structure. One of these, MDL 72222, reported last year", 
has a selectivity similar to the antagonists now described although it is considerably less potent. The Sandoz researchers first synthesized analogues of serotonin and discovered compounds that selectively mimicked its effects on neurones or on smooth muscle. This knowledge was used to synthesize antagonists with selective action on neurones in which the serotonin side-chain nitrogen is incorporated in a tropane ring, as in cocaine (see figure).

Interest in serotonin pharmacology has been particularly strong in the past few years. New generations of antidepressant agents are emerging that act by inhibiting the re-uptake of serotonin after its release from synaptic terminals in brain, thus enhancing its synaptic actions?. It has also been reported recently that novel antianxiety drugs of the buspirone type may act by selective stimulation of serotonin receptors in brain, although the precise category of sites involved remains unclear $^{8}$. Receptors for serotonin of the type that are blocked by the new antagonists have not yet been identified in the central nervous system but their presence there would not be surprising.

As so often in pharmacology, the discovery of novel drugs with selective antagonist actions will undoubtedly allow rapid progress to be made in defining the occurrence and functional significance of a hitherto obscure class of monoamine receptors.

1. Richardson, B. P. Engel, G. Donatsch, P. O. \& Stadler, P. A. Nature 316, 126 (1985)

2. Gaddum, J. H. \& Picarelli, Z. P. Br. J. Pharmac. 12, 323 (1957).

3. Peroutka, S. J. \& Snyder, S. H. Molec. Pharmac. 16, 687 (1979)

4. Leysen, J. E. et al. Neuropharmacology 23, 1493 (1984).

5. Fozard, J. R., Mobarok Ali, A. T. M. \& Muscholl, E. Br J Pharmac. 61, 499 (1977).

6. Fozard, J. R. N. S. Arch. Pharmac. 326, 36 (1984).

7. Blier, P. \& de Montigny, C. J Neurosci. 3, 1270 (1983)

8. Traber, J. et al. Brain Res. Bull. 12, 741, (1984).

Leslie L. Iversen is Director of the Merck, Sharpe \& Dohme Neurosciences Research Centre, Harlow, Essex CM20 2QR, UK.

\section{Surface structure}

\section{$\mathrm{X}$-ray reflection from liquids}

\section{from Stuart A. Rice}

IN AN important paper in Physical Review Letters, A. Braslau et al. report measurements of the surface roughness of water deduced from the scattering of $\mathrm{X}$ rays reflected from the surface'. To do so, they have introduced a new way to interpret the reflectivity data. This and related advances are beginning to revolutionize the understanding of liquid surfaces.

For two decades there has been a steady stream of improvements in the scope and sensitivity of techniques with which to study the atomic and electronic structures of surfaces. Many of these techniques use electrons as probes, or detect electrons, or both. Unfortunately, because electrons interact very strongly with the atomic charge distribution, the interpretation of electron-scattering data usually requires removal of the contributions to the signal from multiple scattering processes, which cannot at present be carried out for liquid surfaces. Unlike electrons, $\mathrm{X}$ rays are weakly scattered by the atomic charge distribution, and two X-ray techniques have recently been introduced to study surfaces: X-ray reflectivity as a function of angle of incidence, and grazing incidence $\mathrm{X}$-ray diffraction. Both can be used to study liquids as well as solids.

That $\mathrm{X}$ rays will be totally reflected from a condensed medium if the angle of incidence is less than some critical value was predicted and first demonstrated by A.H. Compton in the $1920 \mathrm{~s}^{2}$. The thrust of the work in the following decade was concerned with the determination of the refractive index of matter at X-ray frequencies, assuming the sample to be homogeneous up to a planar surface, treated as a geometrical discontinuity. It was not until 1954 that L.G. Parratt suggested inverting the analysis and interpreting $\mathrm{X}$ ray reflectivity as a function of angle of incidence via models of the inhomogeneous surface-density distribution ${ }^{3}$. This suggestion lay dormant for twenty years, until B. C. Lu and S. A. Rice adapted it to provide information about the surface structure of liquid mercury. Other applications $s^{5,6}$ are rare.

$\mathrm{X}$-ray reflectivity as a function of angle of incidence is sensitive to the distribution of density perpendicular to the surface; the greater the range of incident angles for which measurements can be made, the more accurate will be the inferences drawn about the surface density profile. The dynamic range required for such experiments is large - of the order of $10^{7}$ to $10^{8}$ - so synchrotrons are the favoured $\mathrm{X}$-ray sources. The data can be interpreted by two means. In one, a model of the surface-density distribution is assumed and the predicted X-ray reflectivity is compared with that observed. Alternatively, the data can be interpreted in terms of some model-independent parameter that is characteristic of the density profile.

It is the second means of interpretation that has been introduced by Braslau et al. They show that the reflectivity data they obtain can be interpreted in terms of the mean square roughness of the surface without prescribing the molecular mechanism by which the roughness is generated. The value they obtain for this measure of the surface density profile is $3.24 \pm 0.05 \AA$. A molecular dynamics simulation of a rigid charge distribution model of water ${ }^{7}$ shows that the density falls smoothly through the liquid-vapour interface with a characteristic width of $3.56 \AA$; the deviation between these values provides one measure of the inadequacy of the model potential used in the molecular dynamics simulation.

As expected, total external reflection of $\mathrm{X}$ rays is accompanied by fluorescence and, very recently, this has been exploited to study the properties of solid and liquid surfaces. Smirnov ${ }^{8}$ has shown that measurement of the fluorescence intensity as a function of angle of incidence of the exciting $\mathrm{X}$ rays can be used to infer the density distribution perpendicular to the surface - in his case, for a film of germanium deposited on glass. In an independent development, Bloch et al. ${ }^{9}$ have used the same kind of measurement to infer the concentration profile of a dissolved polymer in the solution-vapour interface.

To date, the only reported application of grazing incidence X-ray diffraction to a liquid is by $\mathrm{J}$. Als-Nielsen and P. S. Pershan ${ }^{10}$ who measured the interplanar spacing perpendicular to the surface of a smectic-A liquid crystal. The interpretation of the X-ray diffraction pattern in terms of the in-plane distribution of molecules in a liquid-vapour interface is more difficult than for a solid, because removal of the contribution to the X-ray scattering from atoms below the surface requires knowledge of their distribution as a function of density. Whereas the atomic distribution in a crystal substrate is substantially the same up to the outermost plane of atoms, the variation in distribution in the 2-3-atom thick, inhomogeneous liquid-vapour interface is important. Nevertheless, guided by computer simulations, it should be possible to obtain useful information about the inplane distribution of atoms and molecules in a liquid-vapour interface.

The availability of powerful tunable $X$ ray sources is likely greatly to increase our information base and understanding of liquid surfaces. A few of the possibilities we can expect in the next few years are studies of the structure of films supported on liquids and of phase transitions in those films; measurements of the influence of oxidation on the structure of the liquid surface; and investigations of the spatial distribution associated with the excess surface concentration of a component of a mixture.

\footnotetext{
1. Braslau, A., Deutsch, M., Pershan P.S., Weiss, A.H. Als-Nielsen, J. \& Bohr, J. Phys. Rev. Lett. 54, 114 (1985) 2. Compton, A.H. Bull. natn. Res. Coun. (US) 20, 48 (1922) 3. Parratt. L.G. Phys. Rev. 95,359 (1954).

4. Lu, B.C. \& Rice, S.A. J. chem. Phys. 68, 5558 (1978).

5. Sluis, D. \& Rice, S.A. J. chem. Phys. 79, 5658 (1983).

6. Bosio, L. \& Oumezine, M. J. chem. Phys. 80, 959 (1984)

7. Townsend, M.R., Gryko. J. \& Rice, S.A. J. chem. Phys. 82, 4391, (1985)

8. Smirnov. L.A. Opt. Spectrosc. (USSR) 56, 328 (1984).

9. Bloch, J.M. et al. Phys. Rev. Lett. 54, 1039 (1985).

10. Als-Nielsen. J. \& Pershan, P.S. Nucl. Instrum. Meth. 208, 545 (1983).
}

Stuart A. Rice is Professor of Chemistry at the James Franck Institute, University of Chicago, 4640 Ellis Avenue, Chicago, Illinois 60637, USA 